\title{
Shakespeare et le De Pictura de Leon Battista
} Alberti

Shakespeare and Leon Battista Alberti's theory on linear perspective

Jean-François Chappuit

\section{(2) OpenEdition}

1 Journals

\section{Édition électronique}

URL : http://journals.openedition.org/shakespeare/743

DOI : 10.4000/shakespeare.743

ISSN : 2271-6424

Éditeur

Société Française Shakespeare

Édition imprimée

Date de publication : 1 novembre 2005

Pagination : 55-74

ISBN : 2-9521475-1-5

\section{Référence électronique}

Jean-François Chappuit, «Shakespeare et le De Pictura de Leon Battista Alberti », Actes des congrès de la Société française Shakespeare [En ligne], 22 | 2005, mis en ligne le 01 janvier 2007, consulté le 01 mai 2019. URL : http://journals.openedition.org/shakespeare/743 ; DOI : 10.4000/shakespeare.743 


\section{Shakespeare et l'Europe de la Renaissance}

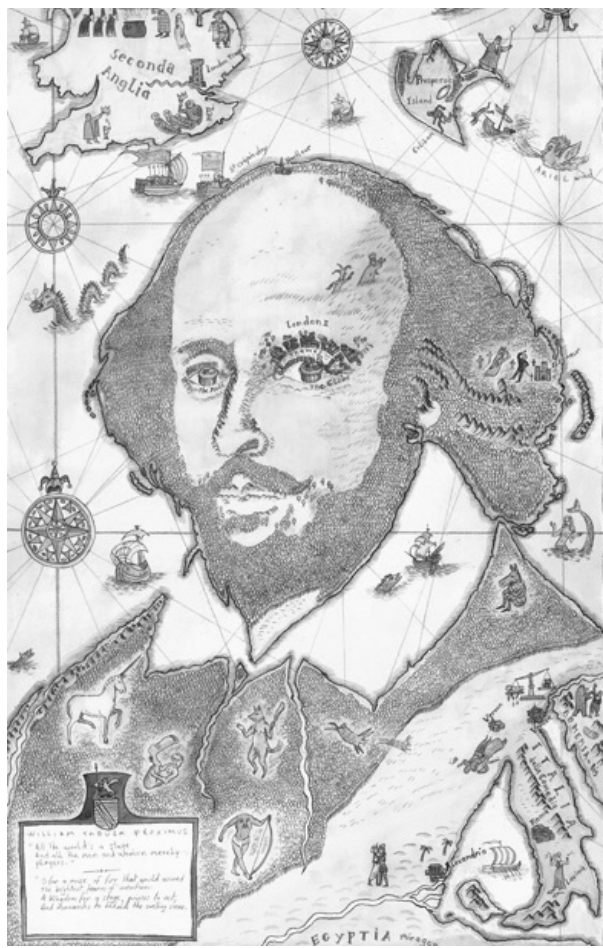

a ctes du Congrès

organisé par la

SOCIÉTÉ FRANÇAISE SHAKESPEARE

les 11,12 et 13 mars 2004

textes réunis par

Pierre KAPITANIAK

sous la direction de

Yves PEYRÉ 


\title{
COMITÉ SCIENTIFIQUE :
}

\author{
Margaret Jones-Davis \\ Jean-Marie Maguin \\ Yves Peyré \\ Pierre Kapitaniak
}

COUVERTURE :

Edouard Lekston, William Tabula Proximus, 2004

conception graphique et logo

Pierre Kapitaniak

CC 2004 Société Française Shakespeare

Institut du Monde Anglophone

Université de Paris III - Sorbonne Nouvelle

http:// univ-montp3.fr/SFS/

5 rue de l’École de Médecine

75006 Paris

ISBN 2-952 1475-1-5

Tous droits de traduction, de reproduction et d'adaptation réservés pour tous les pays 


\title{
S HAKESPEARE ET LE DE PICTURA DE LEON B ATTISTA AlbERTI
}

\author{
Jean-François CHAPPUIT
}

\begin{abstract}
La Renaissance se distingue à la fois par la redécouverte des textes de l'Antiquité et par un nouvel intérêt pour la représentation de l'espace en trois dimensions. Les voûtes, les dômes et les murs des bâtiments construits à l'époque sont souvent couverts de représentations artistiques qui permettent à l'œil, pour ainsi dire, de voir audelà du support naturel. En Angleterre, après un timide début, les nouvelles théories gagnèrent en influence vers la fin du XVle siècle. Or, les poètes ont toujours été comparés à des peintres. Cette comparaison se trouve déjà dans La Poétique d'Aristote (Chap. 25) et au chapitre 4 il affirme que l'homme a une tendance naturelle à représenter le monde qu'il habite et qu'il y trouve du plaisir. La transformation soudaine de l'art dramatique entre Everyman et le théâtre Elisabéthain serait-elle due au nouvel intérêt pour la perspective linéaire issue de la Renaissance en Europe ? Je me propose d'explorer les liens possibles entre le De Pictura (1435) d'Alberti et la production dramatique de Shakespeare.
\end{abstract}

Shakespeare and Leon Battista Alberti's theory on linear perspective Along with the rediscovery of texts from Classical Antiquity, the Renaissance in Europe witnessed a new interest in three-dimensional space in artistic representation. Many of the vaults, domes and walls of the buildings built during that period were covered with paintings that, so to speak, gave the beholder the possibility to see beyond them. In England, after a slow start, the new artistic theories became increasingly important in the late $16^{\text {th }}$ century. Now, poets have always been compared to painters. Aristotle already uses this simile in Poetics (chap. 25.) and in chapter 4 he asserts that man is naturally inclined at representing the world around him and that he finds pleasure in it. Could Renaissance interest in linear perspective explain the sudden change, in England, between Everyman and Elizabethan drama ? In this paper I shall explore the possible links between Alberti's De Pictura (1435) and Shakespeare's plays.

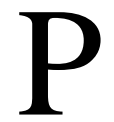

ar la maîtrise de la perspective, les artistes de la Renaissance créaient l'apparence de la réalité. Quel statut accordaient-ils à cette mimèsis? N'était-elle qu'une illusion, voire une tromperie, ou bien avait-elle une réalité objective ? En était-il de même pour la poésie puisque, selon Horace, «ut pictura, poèsis» (Ars poetica, v. 361) ? La critique moderne, et notamment Roland Barthes, nie toute réalité à la mimèsis et lui substitue la structure du langage. Cependant, la perspective ne forme-t-elle pas en elle-même une structure de laquelle la représentation tirerait son statut de réalité ?

L'analogie entre peinture et poésie est encore courante à l'époque élisabéthaine. Elle se trouve incluse dans la définition que Sir Philip Sidney donne dans son Apology for Poetry (1595): "Poesy therefore is an art of imitation, for so Aristotle termeth it in his word mimesis that is to say, a representing, counterfeiting, or figuring forth - to speak metaphorically, a speaking picture - with this end, to teach 
and delight ${ }^{1} \gg$. J'utiliserai le De Pictura (1435) en référence à cette tradition, non pour prouver une quelconque nouvelle influence sur Shakespeare mais pour aborder son théâtre par le biais de la théorie exposée par $\mathrm{Alberti}^{2}$. Ce dernier fit autorité pendant toute la Renaissance. Shakespeare a-t-il réutilisé à son profit les dogmes de l'art pictural de son époque, et avec les mêmes conséquences quant à la mimèsis ${ }^{3}$ ? Cependant, avant d'examiner cette question j'apporterai quelques précisions sur l'analogie entre peinture et poésie.

Selon Plutarque, dans De Gloriam Atheniensium, Simonide de Céos, poète lyrique grec né au VI ${ }^{\mathrm{e}}$ siècle avant Jésus-Christ, évoqua le premier cette analogie :

Or Simonide appelle la peinture une poésie muette et la poésie, une peinture parlante. De fait, les actions que les peintres représentent dans leur accomplissement sont racontées et décrites dans leur achèvement par les ouvrages littéraires. Si traitant les mêmes thèmes, les uns utilisent des couleurs et des figures, les autres des mots et des locutions, s'ils diffèrent par le matériau et les procédés de leur imitation, le but reste le même dans les deux cas, et le meilleur historien est celui qui traite son récit comme une peinture en mettant en scène des événements pathétiques et des personnages 4 .

Donc, si peintre et poète diffèrent quant aux méthodes de mise en œuvre de la mimèsis, ils partagent le même objectif en ce qui concerne le récit, la diégèsis. C'est donc sur ce dernier concept que Plutarque justifie l'analogie.

Dans The Curious Perspective, Ernest Gilman revient sur la validité de cette comparaison entre deux activités artistiques dont les moyens d'expression sont si différents ${ }^{5}$. Au XVIII ${ }^{\mathrm{e}}$ siècle, poursuit-il,

\footnotetext{
${ }^{1}$ Sir Philip Sidney, An Apology for Poetry, éd. Geoffrey Shepherd, 3e éd. Augmentée, éd. R. W. Maslen, Manchester, Manchester University Press, 2002, p. 86. Toutes les citations renverront à cette édition.

${ }^{2}$ Leon Battista Alberti, De la Peinture (De Pictura, 1435), trad. Jean Louis Schefer, intro. Sylvie Deswarte-Rosa, Paris, Macula, Dédale, 1992. Toutes les références au De Pictura renverront à cette édition.

${ }^{3}$ Ce thème fit l'objet de deux colloques, l'un en 1982, l'autre en 1983. Voir Marie-Thérèse Jones-Davies (dir.), Vérité et Illusion dans le théâtre au temps de la Renaissance, Paris, Touzot, 1983.

${ }^{4}$ Plutarque, De Gloria Atheniensium, 3, 346 f, éd. J.-C. Thiolier, Paris, Presses de l'Université de Paris-Sorbonne, 1985, p. 40-41.

${ }^{5}$ Ernest B. Gilman, The Curious Perspective: literary and pictorial $w$ it in the seventeenth century, New Haven (Conn.), Yale University Press, 1978, p. 3. Gilman cite la traduction de
} 
Lessing, dans son Laocoon, ou Des frontières de la peinture et de la poésie (1766), assigne deux champs distincts aux deux activités artistiques, le temps pour la poésie ; l'espace pour l'art visuel. Pourtant, Lessing ne rejette pas entièrement les propos de Simonide, mais les juge vagues ${ }^{6}$. Il en attribue l'origine au sensualisme : «Le premier qui compara peinture et poésie fut un homme d'une grande finesse de goût qui sentait que ces deux arts produisaient en lui les mêmes effets. ${ }^{7}$ »

C'est dans le même esprit que, pour Ernest Gilman, l'analogie entre peinture et poésie se justifie en terme d'expérience. Cette dernière se compose de deux phases, la perception et l'appréhension. Ce point de vue s'inscrit dans le cadre général de la critique structuraliste. L'œuvre d'art n'est que mimèsis; le sens revient à la diégèsis et il est l'affaire de l'observateur. La perspective linéaire est ce qui permet cette expérience. En elle-même, elle représentait à l'époque une façon beaucoup plus dynamique d'appréhender le monde qu'au cours des siècles passés. Ainsi, Ernest Gilman attire-t-il l'attention sur les nombreuses avancées tant pratiques que conceptuelles qui furent réalisées grâce à la maîtrise de la perspective.

Chez Shakespeare, il relève plusieurs références à l'art pictural et à la perspective. Il cite la description du tableau de la chute de Troie dans The Rape of Lucrece, (v. 1366-1463) et les propos d'Enobarbus pour décrire l'arrivée de Cléopâtre sur le fleuve Cydnus (II.2.194-221). J'ajouterai la référence implicite à Nicholas Hilliard par le biais des portraits que la reine Gertrude et Hamlet portent autour du cou : «Look here upon this picture, and on this / The counterfeit presentment of two brothers » (III.iv.52-53). Le mot «presentment» renvoie à la fonction que la Renaissance attribuait à l'art pictural, c'està-dire rendre présent ce qui est absent. D'autre part, Hilliard publia son Art of Limning vers 1600. Ernest Gilman indique aussi que différents objets amusants furent inventés par les peintres. Un de ceuxci consistait en une surface plane plissée avec, sur chaque coté du pli, la partie d'une représentation différente de façon à voir une image complète en regardant la plissure soit d'un coté soit de l'autre. Selon

Sir Thomas Hoby: «Painting is dumme Poesie, and Poesie a speaking picture». Sir Thomas Hoby traduisit en anglais le Livre du Courtisan de Castiglione, en 1561.

${ }^{6}$ Gotthold Ephraïm Lessing, Laocoon, ou Des frontières de la peinture et de la poésie, trad. Courtin (1866) revue et corrigée, avant propos de Hubert Damisch, «Savoir sur l'Art », Paris, Hermann, 1990, p. 42.

${ }^{7}$ Ibid., p. 41. 
Ernest Gilman Shakespeare avait ce type d'objet en tête lorsque Cléopâtre s'écrie : "Though he be painted one way like a Gorgon, / The other way's a Mars» (II.v.116-118). Gilman mentionne aussi Twelfth Night, lorsque Orsino voit Viola et Sébastien côte à côte : "One face, one voice, one habit, and two persons, / A natural perspective, that is and is not $»($ v.i.200-201).

Cependant, son argumentation à propos de la façon dont Shakespeare utilise le concept de perspective repose principalement sur deux textes. Le premier est tiré du sonnet $n^{\circ} 24$ :

Mine eye hath played the painter and hath stelled, Thy beauty's form in table of my heart, My body is the frame wherein 'tis held, And perspective it is best painter's art.

Sans prétendre trouver une quelconque influence, il me semble néanmoins digne d'intérêt de relever que, dans ce sonnet, le mot «table » évoque l'« intersecteur» ou «voile»dont Alberti se sert pour tracer le contour de ce qu'il peint :

Il est ainsi fait : c'est un voile de fils très fins, tissé lâche, teint d'une couleur quelconque, divisé au moyen de fils plus épais en autant de bandes de carrés qu'on voudra et tendu sur un cadre. Je le place entre le corps à représenter et l'œil, de façon que la pyramide visuelle pénètre à travers les jours du voile. (II, 31, p. 147)

Quant au cadre, «frame»dans le sonnet, il remplit la même fonction que ce que Alberti appelle la fenêtre c'est-à-dire le quadrilatère à partir duquel les lignes de fuite sont déterminées :

Je trace d'abord sur la surface à peindre un quadrilatère de la grandeur que je veux, fait d'angles droits, et qui est pour moi une fenêtre ouverte par laquelle on puisse regarder l'histoire, et là je détermine la taille que je veux donner aux hommes dans ma peinture. (I, 19, p. 115)

Enfin, le verbe «to stell», "délinéer», indique exactement ce que $l^{\prime} \ll$ intersecteur » permet de faire.

Le second texte important est le passage où, dans Richard II, Bushy console la reine :

Each substance of a grief hath twenty shadows

Which show like grief itself but is not so.

For sorrow's eye, glazèd with blinding tears,

Divides one thing entire to many objects -

Like perspectives, which, rightly gazed upon, 


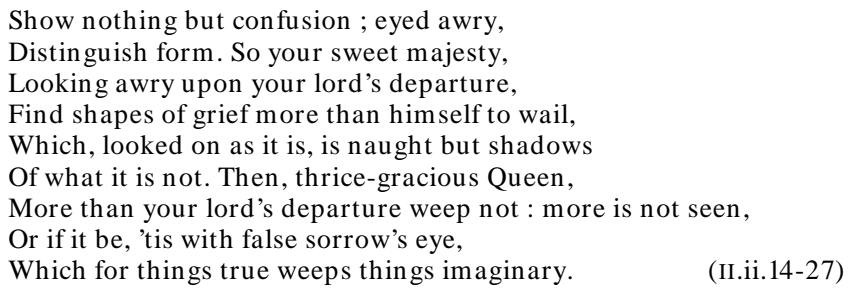

L'argument principal de Gilman s'appuie sur les vers 18 à 20 de ce passage. Il suggère que Shakespeare demande au spectateur de regarder la pièce «de biais» («awry») pour en comprendre le véritable sens. Il reprend le vers du sonnet $24:$ : And perspective it is best painter's art » pour l'appliquer à l'art dramatique et en conclure :

Perspective is best dramatist's art as well: the analogy points to Shakespeare's fundamental concern, beneath whatever emblems and other visual motifs he may call to our intention, with the complex activity of witnessing a play, Shakespeare's viewer is typically asked to see not just the drama, but «through » the dramatist to his « skill» - to the technical bones and sinews of play-wrighting that both support the dramatic illusion and reveal the fragility of its surface ${ }^{8}$.

C'est l'objet même de cette perspective dramatique que je voudrais réexaminer. A-t-elle pour objectif, comme le dit Gilman, d'inviter le spectateur à regarder de biais « awry» pour voir juste «aright », c'est-à-dire percer au jour l'illusion théâtrale ? Ou bien, fait-elle partie de l'œuvre même, comme la perspective dans un tableau est constitutive de ce tableau, pour entraîner le spectateur dans l'expérience même de la perspective et de tout ce qu'elle implique symboliquement ? Je tenterai de répondre à cette question à l'aide des éléments constitutifs de l'art pictural selon Alberti.

L'originalité du De Pictura repose sur une approche scientifique, et notamment mathématique, du traitement de l'espace. L'objectif de l'art pictural, chez Alberti, est l'expression de la vérité issue de la Nature, elle-même source de toute connaissance. Alberti s'appuie également sur la théorie de la perception visuelle héritée de l'optique d'Euclide et le concept qu'il appelle l'historia. J'examinerai en premier lieu la relation entre la Nature et la représentation.

${ }^{8}$ Gilman, op. cit., p. 89. 


\section{Nature et représentation}

Dès le début de son traité, Alberti expose son programme de façon scalaire, sur trois niveaux. Le premier plan est celui des mathématiques: "afin que notre exposé soit plus clair, nous emprunterons tout d'abord aux mathématiciens les éléments qui nous semblent concerner notre sujet» (I, 1, p. 73). À la Renaissance, les mathématiques sont considérées comme la seule science capable d'émettre des certitudes. Les autres sciences ne découvrent que ce qui est probable. Le deuxième niveau est celui de la Nature: «nous traiterons de la peinture en partant des principes mêmes de la nature » (I, 1, p. 73). Cette dernière est la source de toute connaissance. Alberti insiste sur ce point en de nombreux endroits de son traité. Le peintre apprend tout de la Nature. La raison en est simple ; la Nature est ellemême artiste : «Il est d'ailleurs manifeste que la nature même prend plaisir à peindre » (II, 23, p. 143). Le troisième plan est celui de l'art du peintre: «en tout cet exposé je ne parle pas de ces choses en mathématicien mais bien en peintre». La structure de cette introduction est celle des dialogues de Platon dans lesquels, de degré en degré, on en arrive à ce qui est le plus essentiel; dans le De Pictura, c'est le peintre. En inversant l'ordre utilisé par Alberti, nous aboutissons à la proposition suivante : la peinture représente la Nature de façon mathématique, c'est-à-dire certaine. L'introduction de ce traité est un défi lancé à Platon.

En effet, dans La République, le peintre est l'imitateur d'un produit éloigné de trois degrés du réel (X, ii, 599a) tandis que l'Être Suprême est le créateur de ce qui est «essentiel », en l'occurrence, du « lit essentiel». La façon dont Alberti introduit son sujet représente une inversion de ce schéma. Les mathématiques sont un instrument abstrait, la Nature un matériau et sur le tableau s'exprime ce qui est « essentiel », c'est-à-dire de l'ordre des «Idées ». Il ne s'agit pas d'une opposition à Platon, mais d'une appropriation du platonisme dans une perspective chrétienne. L'artiste est lui-même démiurge parce qu'il a été créé à l'image de Dieu (Genèse, 1, 26). L'homme est d'essence divine par son intelligence. Chez Alberti, le peintre est le créateur de membres et de corps pour finalement aboutir à l'historia qui est «le dernier degré d'achèvement de l'œuvre» (II, 35, p. 159). À une échelle plus modeste, le peintre est comme Dieu, le créateur de mondes. Une conception similaire se trouve au siècle suivant, chez Sir Philip Sidney 
dans son Apology for Poetry. Sidney s'appuie d'abord sur l'étymologie du mot «poète » : «It cometh of this word poiein, which is "to make": wherein I know not whether by luck or wisdom, we Englishmen have met with the Greeks in calling him "a maker" "(p. 84). Le poète est véritablement l'image de Dieu, Son double; c'est la raison pour laquelle il domine toutes les autres sciences. Plus loin dans l'Apology, Sidney reprend ce concept pour faire le parallèle entre «the Maker» et «the maker» :

Neither let it be deemed too saucy a comparison to balance the highest point of man's wit with the efficacy of nature ; but rather give right honour to the heavenly Maker of that maker, who having made man to His own likeness, set him beyond and over all the works of that second nature: which in nothing he showeth so much as in Poetry, when with the force of a divine breath he bringeth forth far surpassing her doings, with no small argument to the incredulous of that first accursed fall of Adam : since our erected wit maketh know what perfection is, and yet our infected will keepeth us from reaching unto it. (Apology, p. 85)

L'expression «our infected will» renvoie aux passions humaines. Sidney évoque ici ce que dit saint Paul dans l'Épître aux Romains: «Car je sais qu'en moi - je veux dire dans ma chair - le bien n'habite pas; vouloir le bien est à ma portée, mais non pas l'accomplir, puisque le bien que je veux, je ne le fais pas et le mal que je ne veux pas, je le fais » (7, 18-19). Le poète exprime la conscience morale de l'humanité ; il ravive tout ce que l'homme a perdu depuis la Chute. Mais il n'y a pas d'opposition entre Nature et art; au contraire, l'art est l'accomplissement de ce qui est en puissance dans la Nature, son raffinement. Sidney souligne cet aspect de l'art de façon très explicite : "Nature never set forth the earth in so rich a tapestry as divers poets have done [...] Her world is brazen, the poets deliver a golden » (p. 85). Cette vue sur l'art se trouve déjà chez Alberti. L'observation exacte de la Nature est la première étape sur le chemin qui conduit à l'accomplissement de l'œuvre: «Aussi est-ce la grâce et la beauté que l'on doit d'abord rechercher dans cette composition des surfaces. Et quelle que soit la façon dont nous y parvenons, je ne vois pas de chemin plus sûr que d'observer la nature même et de regarder longtemps et avec soin comment la nature, en artiste admirable, a composé les surfaces et les membres les plus beaux. Il faut donc s'attacher à l'imiter en cela avec tout le soin et la réflexion possibles...» (II, 35, p. 159). L'art représente un point de départ vers la Vérité. 
Enfin, l'usage qu'Alberti fait du miroir révèle une nouvelle fois de quelle façon il renverse l'enseignement de Platon. Dans la République, le miroir est un objet de mensonge grâce auquel celui qui le tient prétend être l'égal du Tout Puissant (x, i, 596c-e). Chez Alberti, le miroir met en évidence ce qui est faux; en d'autres termes il est un moyen d'accès vers la Vérité : « le miroir sera un très bon juge. [...] toute erreur du peintre est accusée dans le miroir. Ce qui est emprunté à la nature doit donc être corrigé par le jugement du miroir » (II, 46, p. 195). Ces lignes nous renvoient au célèbre passage où Hamlet conseille aux acteurs «to hold as 'twere the mirror up to nature ». Cette fonction est celle de l'art dramatique dans son ensemble et cela, précise Hamlet, depuis les origines : «For anything so o'erdone is from the purpose of playing, whose end, both at the first and now, was and is to hold as 'twere the mirror up to nature » (Hamlet, III.ii.16-36). L'ensemble constitue un abrégé d'art poétique. Le premier précepte concerne l'instrument même de l'art, l'intelligence : «let your own discretion be your tutor». Cette injonction fait écho aux propos de Sidney pour qui les véritables poètes « have no law but wit » (Apology, p. 86). Deuxième point, la nature doit être copiée avec exactitude. Cet aspect rejoint ce que dit Alberti dans son traité et ce qui est généralement accepté à la Renaissance. Troisièmement, Shakespeare, comme Alberti, fait du miroir un vecteur de vérité et utilise la notion d'« empreinte » pour décrire la représentation : «to show [...] the very age and body of the time his form and pressure ». Ainsi, entre le monde et sa représentation il existe un lien organique sur lequel nous reviendrons. L'imitation exacte de la nature ne constitue pas une simple répétition mais un dépassement et cela sur le plan de l'intelligence. C'est le dernier point mentionné par Hamlet : l'objet de l'art dramatique est l'imitation de l'humanité, c'est-à-dire d'une réalité qui dépasse l'individu et que ce dernier ne peut comprendre que par son intelligence, « his wit » pour Sidney.

Cependant, il semble entendu que l'art, quel qu'il soit, repose sur la perception oculaire car le poète ou le peintre crée une vision nouvelle. L'œil est donc l'organe privilégié de tout art. Dans la réflexion d'Alberti, le rapport au monde et à l'œuvre s'appuie sur la théorie du fonctionnement de l'œil héritée de l'Optique d'Euclide. 


\section{Représentation et perception}

Le rôle attribué à l'œil est important parce qu'il détermine le rapport qui existe entre le sujet et l'œuvre et, par extension, entre le sujet et le monde. À la Renaissance, il est admis que la perception visuelle est constituée de rayons. Alberti fait sienne cette théorie : «les surfaces sont mesurées par certains rayons, véritables ministres de la vue, qu'ils [les philosophes] appellent rayons visuels parce que c'est par eux que les simulacres des choses s'impriment dans la vue»(I, 5, p. 81). Ces rayons sont doués de force physique qui les fait s'attacher à tout ce qu'ils touchent. Le second aspect de cette théorie concerne l'endroit où se concentrent les rayons visuels. Deux opinions s'opposent et que Alberti mentionne clairement. Selon la première, les rayons se rejoignent sur la surface de l'œil qui, ainsi, joue le rôle de miroir. Il y aurait, alors, un face-à-face entre l'œil et ce qu'il voit ou entre le sujet et le monde. Cette théorie du «miroitement» de l'œil est suggérée dans les propos que Bushy adresse à la reine dans Richard II. Cependant, chacun sait que le raisonnement de Bushy est fallacieux. Dans la deuxième théorie, les rayons visuels se concentrent en un point à l'intérieur de l'œil. C'est celle qu'Alberti adopte (I, 5, p. 83). La différence est importante car, dans ce cas, la perception est une appropriation ou, pour reprendre un concept emprunté à Nicolas de Cues, un enveloppement. L'opinion d'Alberti était, en fait, déjà manifeste avec la notion d'impression qui se trouve dans le texte cité plus haut (I, 5, p. 81). Les rayons visuels impriment au fond de l'œil, comme dans de la cire, les contours autour desquels ils se sont attachés. Le rapport ainsi suggéré est un rapport d'union organique. Cette façon de comprendre la perception visuelle correspond au besoin de dépassement des oppositions scolastiques qui nourrissait le mouvement intellectuel de la Renaissance.

Les deux théories évoquées ci-dessus sont exploitées par Shakespeare. Celle de l'œil comme miroir, nous l'avons vu, est suggérée dans les paroles adressées à la reine par Bushy. Je n'y reviendrai pas. Un autre exemple se trouve dans la scène entre Brutus et Cassius dans Julius Caesar:

CASsiUs. Tell me, good Brutus, can you see your face?

BRUTUS. No, Cassius, for the eye sees not itself

But by reflection, by some other things. 
CASsius. 'Tis just ;

And it is very much lamented, Brutus,

That you have no such mirrors as will turn

Your hidden worthiness into your eye,

That you might see your shadow.

(I.ii.53-60)

L'offre de Cassius représente une image de repli de la vision sur ellemême et donc d'enfermement et d'aveuglement. Elle correspond à la logique scolastique qui oppose des contraires pour mieux les comprendre. La réponse de Brutus va dans le même sens et indique de quelle façon le personnage s'engage dans l'erreur. En effet, la phrase «for the eye sees not itself» rappelle le problème de la connaissance de soi débattu par Plotin dans le traité $n^{\circ} 49$ : «Est-ce que ce qui se pense soi-même doit être différencié pour que, contemplant par un de ses éléments les autres, on puisse en ce sens affirmer de lui qu'il se pense lui-même, parce que ce qui est absolument simple ne peut se tourner vers soi-même et vers la saisie de soi ? ${ }^{9}$ Plotin répond que la connaissance de soi se fait par l'intellection et que, comme la lumière est lumière d'elle-même, l'intellection est intellection d'elle-même. De même, l'œil est ce qu'il voit et, inversement, ce qui est vu constitue l'œil. Shakespeare suggère cela par les propos de Brutus qui, en définitive, voit tout ce qu'il y à voir :

Into what danger would you lead me, Cassius,

That you would have me seek in to myself

For that which is not in me?

Cependant, la seconde théorie est aussi présente dans cet échange par le biais de la préposition «into » (55). La perception pénètre l'œil et se fait en lui. Cet aspect est repris plus bas dans la même scène lorsque Brutus dit à Cassius : "Set honour in one eye and death i'th'other, / And I will look on both indifferently» (I.ii.88-89). Il faut comprendre qu'il s'agit des yeux de Brutus et non de Cassius: «Placez l'honneur dans un de mes yeux et la mort dans l'autre ». L'œil n'est pas extérieur à la Nature comme le miroir l'est implicitement dans le célèbre passage de la République de Platon (X, i).

Cette théorie de la perception visuelle a pour conséquence d'attribuer à toute représentation un statut de réalité objective qui est différent de la réalité physique mais qui en partage certains éléments et

${ }^{9}$ Plotin, Traité 49, v, 3, éd. et trad. Bertrand Ham, Paris, Éditions Du Cerf, 2000, p. 47. 
notamment celui de l'«impression ». Dans Ham let, la passivité du roi et de toute la cour devant le mime doit se comprendre en relation avec les conseils d'Hamlet aux acteurs. La pièce représentée doit être la forme et la marque du siècle. Il faut alors compléter l'argumentation : ainsi elle marque à son tour celui qui la regarde par l'«impression » visuelle. Le mime n'est pas ce miroir dont parle Hamlet (III.ii.22-24). I1 n' « impressionne » ni le roi ni la cour ; en revanche, la pièce, miroir du crime perpétré, y parvient. Chez Sidney nous avons vu que le poète est créateur sur le modèle du Grand Créateur. Ainsi, ce que produit le poète est réel, d'une part en raison de l'origine de sa création, c'est-àdire l'«Idée »; d'autre part en raison des conséquences pratiques de l'œu vre, à savoir son enseignement :

Neither let this be jestingly conceived, because the work of the one be essential, the other in imitation or fiction; for any understanding knoweth the skill of the artificer standeth in that Idea or fore-conceit of the work, and not in the work itself. And that the poet hath that Idea is manifest, by delivering them forth in such excellency as he hath imagined them. Which delivering forth also is not wholly imaginative, as we are wont to say by them that build castle in the air; but so far substantially it worketh, not only to make a Cyrus, which had been but a particular excellency as Nature might have done, but to bestow upon the world to make many Cyruses, if they will learn aright why and how that maker made him. (Apology, p. 85)

En d'autres termes, Sidney fonde la réalité objective de l'œuvre d'art sur l'existence de Dieu. L'œuvre est objectivement réelle parce qu'elle est vecteur de connaissance et, par ce moyen, la voie d'accès à la jouissance de l'essence divine perdue lors de la Chute (Apology, p. 88). Ainsi, toutes les sciences sont-elles subalternes («serving sciences»), parce qu'elles n'ont que leur propre sujet comme intérêt, alors que la poésie vise la science des sciences, ce que Sidney nomme architectonike. Cette dernière se trouve en l'homme lui-même, ce qui fait de lui le principe même de l'architecture universelle : « the mistress knowledge by the Greeks called architectonike, which stands (as I think) in the knowledge of man's self, in the ethic and politic consideration, with the end of well-doing and not of well-knowing only » (Apology, p. 88). De tout cela se dégage la notion selon laquelle les différents plans de réalité, pour les appeler ainsi, ne s'opposent pas mais sont liés par des correspondances et des similarités, des «sympathies» qui permettent la circularité de l'un à l'autre. Cela correspond exactement à ce que permet la symétrie. En bref, l'art est 
l'expression de la structure universelle créée par Dieu, le Grand architecte. D'une structure à l'autre le rapport est symétrique; nous verrons comment plus bas. Ainsi, après avoir comparé le philosophe et le poète, Sidney conclut :

for whatever the philosopher saith should be done, he [the poet] giveth a perfect picture of it in some one by whom he presupposeth it was done, so as he coupleth the general notion with the particular example. A perfect picture I say, for he yieldeth to the powers of the mind an image of that whereof the philosopher bestoweth but a wordish description, which doth neither strike, pierce, nor possess the sight of the soul so much as that other doth. (Apology, p. 90)

Relevons la correspondance entre poésie et art pictural. La poésie ou l'art se voit par la vue de l'âme; ce qui correspond à la visio intellectualis, la nouvelle perspective intellectuelle dont Nicolas de Cues est l'initiateur.

Architecte, Alberti n'emploie pourtant pas le mot architectonike dans le De Pictura; mais le mot historia fait implicitement référence à cette notion parce qu'il désigne, je le crois, un réseau de perceptions.

\section{Perception et historia}

L'historia est ce qui se voit par la «fenêtre », c'est-à-dire le cadre à partir duquel partent les lignes de la perspective (I, 19). Au livre II, Alberti indique de quelle façon l'historia «constitue le dernier degré d'achèvement de l'œuvre du peintre» (II, 35, p. 159). En note, JeanLouis Scheffer précise qu'il s'agit d'un des concepts majeurs du $D e$ Pictura :

Ni le terme d'histoire, ni celui d'anecdote ou de sujet ne convient tout à fait. L'historia est l'objet même de la peinture qui résulte d'une invention (le sujet, qui peut faire l'objet d'une narration ou d'une description) et d'une composition achevée (agencement des formes, des parties, des corps). On ne peut cependant exclure cette acception simple: le programme «réaliste» d'Alberti exige que la peinture montre et raconte. Dans sa définition la plus formelle, l'historia est un agencement de parties (de corps, de personnages, de choses) doté de sens.

Relevons les derniers mots : un agencement de parties doté de sens. Il faut faire crédit à Alberti de ne pas s'être enfermé dans la seule perspective du point de fuite. L'historia fait référence à un réseau de 
rapports entre différents personnages représentés. Ce réseau exprime ce que Aristote dénomme muthos. Si, grâce au point de fuite, l'espace est organisé autour des personnages, ces derniers n'en représentent pas moins chacun un point de vue. L'intérêt du tableau réside dans la combinaison qui résulte de tous ces points de vue. L'organisation de l'espace représente l'univers clos qui est encore accepté à l'époque d'Alberti; mais au sein de cet univers les «perspectives» sont multiples et complexes. À mon sens, le même principe organisationnel joue dans les pièces de Shakespeare. Dans Hamlet, la référence à un univers clos, limité par « la sphère des fixes », intervient au début et à la fin de la pièce avec la référence au bruit des canons qui part de la Terre pour rebondir au Ciel et redescendre sur Terre. Dans ce cadre, différents personnages représentent chacun une perspective.

Alberti fait aussi de l'homme sont sujet principal parce que la figure humaine lui sert de mesure : «La comparaison se fait donc d'abord avec des choses très connues. Et comme l'homme est pour l'homme la plus connue de toutes les choses, peut-être Protagoras, lorsqu'il disait que l'homme est la mesure et la règle de toute choses, entendait-il que l'on pouvait précisément connaître les accidents de toutes les choses en les comparant à l'homme»(I, 18, p. 113). La référence à Protagoras représente un point de vue relativiste. Quelle est la source du bien fondé des valeurs ; un point de vue humain ou divin ? Shakespeare exploite ce thème en maints endroits et notamment dans Hamlet :

This most excellent canopy the air, look you, this brave o'erhanging, this majestical roof fretted with golden fire - why, it appears no other thing to me than a foul and pestilent congregation of vapours. What a piece of work is man! How noble in reason, how infinite in faculty, in form and moving how express and admirable, in action how like an angel, in apprehension how like a god - the beauty of the world, the paragon of animals! And yet to me what is this quintessence of dust? (II.ii.277281)

Que ce soit sur le ciel ou sur l'homme, Hamlet exprime deux points de vue contradictoires. Ainsi, dans le même personnage coexistent différentes perspectives: le personnage lui-même représente une historia.

Shakespeare emploie le mot history dans cette acception dans Twelfth Night lorsque Orsino s'enquiert de la sœur de Viola déguisée en Césario: «And what's her history?» (II.v.106). Dans sa 
Concordance, Marvin Spevack relève vingt-deux occurrences du mot history dont la plupart ont le sens relevé chez Alberti et notamment dans ces vers prononcés par Warwick à l'intention d'Henri IV dans 2 Henry IV :

There is a history in all men's lives

Figuring the nature of the times deceas'd.

(III.i.80-81)

Ici, Warwick fait bien référence à un agencement de faits à partir duquel, il le dit quelques vers plus bas, Richard II a pu deviner ou lire, avec justesse, la rébellion à venir de Northumberland contre Henri IV : " And by the necessary form of this / King Richard might create a perfect guess » (87-88). Ces vers prennent un sens plus marqué si l'on retient l'acception mathématique des mots «figuring » (81) et «form » (87).

Le mot history désigne aussi un ensemble d'éléments dont il faut déchiffrer le sens dans le sonnet $n^{\circ} 93:$ : In many's looks, the false heart's history/ Is writ in moods and frowns and wrinkles strange.»

Cependant, Shakespeare emploie aussi story dans le sens de history tel que je l'ai défini plus haut. C'est le cas dans Hamlet : "And in this harsh world draw thy breath in pain / To tell my story» (v.ii.281-283). Quel que soit le terme employé, il s'agit de muthos, un récit qui exprime ce que l'homme ne peut comprendre. Ce paradoxe se résout par l'architectonike de la perception.

À la suite d'Euclide la vision, pour Alberti, est constituée de rayons. L'ensemble forme une pyramide visuelle dont le sommet, nous l'avons vu, pénètre jusqu'au fond de l'œil. Il y a un rayon central qui va directement de l'œil au point de fuite et des rayons extérieurs, ceux qui délimitent la pyramide. Ces derniers enveloppent tout ce quils touchent comme des dents dans une bouche: "De plus, les rayons extérieurs embrassant tout le contour de la surface à la manière d'une rangée de dents l'enferment tout entière comme dans une bouche » (I, 7, p. 87). L'œil s'approprie ce qu'il voit. Ces rayons extérieurs entourent le cadre, la «fenêtre» par laquelle l'historia est vue. Ils constituent donc une première pyramide qui va de l'œil au cadre et dont l'aire de ce dernier représente la base. Les lignes de fuite tracées à partir du cadre forment à leur tour une autre pyramide, mais inversée par rapport à la première. Ces deux pyramides sont dans un rapport symétrique, plus précisément de symétrie orthogonale. D’autre part, le 
point de fuite constitue en lui-même le point de référence d'une symétrie centrale si l'on imagine que les lignes de fuite continuent audelà. De cette façon, l'espace organisé par les lignes de fuite est reproduit en opposition « circulaire », c'est-à-dire que ce qui se trouve à droite dans la composition se retrouve à gauche; et ce qui est en haut, en bas.

Cependant, le mot symétrie est à l'origine un terme d'architecture. Les acceptions mathématiques que je viens d'utiliser représentent une spécialisation du mot. Elles ne deviennent courantes qu'au XVIII siècle. Néanmoins, symétrie vient du grec summetria «juste mesure, proportions, symétrie», lui-même dérivé de summetros « de même mesure », « qui a de l'analogie avec». En raison de cette étymologie et de l'intérêt pour l'architectonikè, je soutiendrai que les deux types de symétrie décrites plus haut étaient à l'œuvre dans les champs littéraires et conceptuels bien avant toute application mathématique. Par exemple, le rapport du macrocosme au microcosme représente une symétrie centrale dont la Nature est le centre; si le macrocosme est Dieu, «sphère infinie dont le centre est partout et la circonférence nulle part ${ }^{10} »$. Les mathématiciens définissent la symétrie comme un mouvement circulaire. Ce dernier caractérise le rapport du macrocosme au microcosme et inversement. D'autre part, le concept de symétrie était important dans la rhétorique de Quintilien (v. 30-100), ainsi que chez Cicéron. Dans son introduction au De Pictura, Sylvie Deswarte-Rosa souligne le rôle du De Institutione oratoria dans la composition du traité. Alberti était aussi architecte. Nous lui devons de nombreuses façades dont celle de l'église Santa Maria Novella à Florence. Dans son De Re Aedificatoria, Alberti voit dans le principe de symétrie la loi la plus parfaite de la Nature. Dans la traduction anglaise, la seule que nous avons pu consulter, les traducteurs ont conservé le latin concinnitas, mot de rhétorique qui signifie «symétrie, arrangement symétrique des mots »:

That is why when the mind is reached by way of sight or sound, or any other means, concinnitas is instantly recognized. It is our nature to desire the best, and to cling to it with pleasure. Neither in the whole body nor in its parts does concinnitas flourish as much as it does in Nature herself; thus I might call it the spouse of the soul and of reason.

\footnotetext{
10 Deuxième définition de Dieu dans le Livre des vingt-quatre philosophes, ouvrage hermétique composé au Moyen Âge. Le Livre des Vingt-quatre philosophes, trad. et éd. Françoise Hudry, postface de Marc Richier, Grenoble, Jérôme Million, 1989, p. 95.
} 
It has a vast range in which to exercise itself and bloom - it runs through man's entire life and government, it moulds the whole of Nature. Everything that Nature produces is regulated by the law of concinnitas, and her chief concern is that whatever she produces should be absolutely perfect. Without concinnitas this could hardly be achieved, for the critical sympathy of the parts would be lost. [...] Beauty is a form of sympathy and consonance of the parts within a body, according to definite number, outline, and position, as dictated by concinnitas, the absolute and fundamental rule in Nature ${ }^{11}$.

Dans le théâtre de Shakespeare, les exemples de symétrie se trouvent sur différents plans. Sur celui des énoncés, «fair is foul and foul is fair » dans la première scène de Macbeth représente exactement une symétrie centrale : le premier mot, «fair » se retrouve à la fin de la deuxième phrase; le sens de la première phrase est inversé dans la seconde. L'ensemble forme aussi un palindrome et renforce ainsi la notion de circularité caractéristique de la symétrie. Cet énoncé suggère l'absence d'ancrage stable, de point de vue déterminé pour fixer le sens des valeurs humaines. Au cours de l'été 1583, Giordano Bruno avait donné à Oxford une série de conférences sur les théories de Copernic. Shakespeare avait alors dix-neuf ans. Bruno défendait la thèse copernicienne à l'exception de la sphère des fixes conservée par Copernic. Bruno soutenait que l'espace est illimité; ce qui pose le problème de la localisation du point de référence. Ce dernier n'est plus fixe, mais relatif.

La stichomythie représente aussi une figure de symétrie :

GERTRUDE. Hamlet, thou hast thy father much offended.

HAMLET. Mother, you have my father much offended.

(Ham let, III.iv.9-10)

Dans le vers prononcé par la reine, le «thou» évoque le rapprochement d'elle à Hamlet, tandis que le «thy » de « thy father », son éloignement par rapport au roi décédé. Dans l'énoncé d'Hamlet, le jeu des pronoms a la fonction inverse. Dans l'organisation de l'intrigue, avec les répétitions de scènes ou l'exploitation de personnages en miroir, la notion de symétrie décrit de façon plus parlante la stratégie exploitée par Shakespeare. D'autre part, il faudrait examiner de façon plus précise que je ne peux le faire ici, de quelle façon le concept de symétrie est aussi à l'œuvre dans la structure générale des pièces. Ces

\footnotetext{
${ }^{11}$ Leon Battista Alberti, On the Art of Building in Ten Books, (De Re Aedificatoria), IX, 5, trad. Joseph Rykwert et al., Cambridge (Mass.), The M.I.T. Press, 2e éd., 1989, p. 302-303.
} 
dernières commencent toujours de façon lapidaire; puis l'action s'élargit. Hamlet en donne un bel exemple avec le «Who's there?» (I.i.1) de Barnardo qui est la sentinelle de relève et non celle qui est en faction. L'intrigue se complique ensuite jusqu'à la scène centrale de la pièce dans la pièce à l'acte III. Dans cette première partie, la sphère publique domine. Métaphoriquement, cette première partie correspond à la pyramide visuelle qui va de l'intérieur de l'œil à la «fenêtre». Dans la seconde partie, l'action se restreint à quelques personnages et au destin singulier d'Hamlet. Cette deuxième partie, je la fais correspondre à la pyramide formée par les lignes de fuite tracées à partir de la «fenêtre ». Nous avons bien ainsi la représentation d'un destin individuel dans un cadre général. De cette façon, Shakespeare réalise ce qui fonde la supériorité du poète sur le philosophe, aux yeux de Sidney, parce qu'il « coupleth the general notion with the particular example. A perfect picture I say, for he yieldeth to the powers of the mind an image of that whereof the philosopher bestoweth but a wordish description, which doth neither strike, pierce, nor possess the sight of the soul so much as that other doth» (Apology, p. 90). Enfin, l'annonce de la répétition de l'histoire qui vient d'être jouée évoque la symétrie centrale dont j'ai parlé plus haut, l'existence d'un espace audelà du point de fuite. Elle est fréquente. Dans Othello, le dernier vers l'évoque (v.ii.350-351). Dans Hamlet, elle est faite par Horatio (v.ii.315-316). Cette symétrie permet la circularité entre le temps de l'action représentée, et celui de la représentation. L'évocation d'une redite annonce que ce qui vient d'être représenté se passera de nouveau. C'est suggérer à la fois la proximité et l'éloignement. Passé et présent ne cessent de communiquer.

\section{Conclusion}

Dans les Ambassadeurs (1533) de Hans Holbein le Jeune, lorsque les ambassadeurs sont visibles, le crâne ne l'est pas mais, lorsque l'angle visuel permet de le voir, il devient impossible de distinguer les deux hommes. La scène est située dans Westminster Abbey comme l'atteste le dallage. Les deux personnages sont dans un rapport de symétrie axiale par leur position et aussi par leurs fonctions. Jean de Dinteville appartient au monde séculier, son ami, Georges de Sèlve, au monde ecclésiastique. Leur représentation et celle du crâne sont dans un 
rapport de symétrie centrale. Un troisième élément, caché, se tient derrière l'épaisse tenture. Puisque nous sommes à Westminster Abbey, c'est la divinité, toujours présente, centre et circonférence. Ainsi, chaque «perspective» exclut l'autre, mais un élément reste toujours présent, englobant, c'est la présence de Dieu. Shakespeare multiplie aussi les perspectives et chacune d'entre elles exclut les autres parce que perception, composition et historia forment, comme sur la toile du peintre, un ensemble inséparable.

Montaigne nous offre un autre exemple d'application de la perspective et de la symétrie dans l'essai «De l'amitié » (I, xxviii), publié en 1580 pour la première fois :

Considérant la conduite de la besongne d'un peintre que j'ay, il m'a pris envie de l'ensuivre. Il choisit le plus bel endroit et milieu de chaque paroy, pour y loger un tableau élabouré de toute sa suffisance; et, le vuide tout autour, il le remplit de crotesques, qui sont peintures fantasques, n'ayant grace qu'en la variété et estrangeté. Que sont-ce icy aussi, à la vérité, que crotesques et corps monstrueux, rappiecez de divers membres, sans certaine figure, n'ayants ordre, suite ny proportion que fortuité ?

Desinit in piscem mulier formosa superne. [C'est le corps d'une belle femme, que finit une queue de poisson, Horace, Art poétique, 4.]

Je vay bien jusques à ce second point avec mon peintre, mais je demeure court en l'autre et meilleure partie: car ma suffisance ne va pas si avant que d'oser entreprendre un tableau riche, poly et formé selon l'art. Je me suis avisé d'en emprunter un d'Estienne de la Boitie, qui honorera tout le reste de cette besongne ${ }^{12}$.

À cette époque, parler de tableau sur un mur c'est immanquablement faire référence à la perspective. Alberti, dans son traité, désigne le mur comme support naturel pour un tableau (II, 31, p. 149). Deuxièmement, lorsqu'il mentionne le milieu du mur, Montaigne renvoie le lecteur à la notion de circularité et à celle de point de fuite. La notion de circularité est renforcée avec l'indication des grotesques qui encerclent l'œuvre centrale. Elle se retrouve en d'autres endroits de l'essai et notamment en son centre avec la célèbre formule «Par ce que c'estoit luy; par ce que c'estoit moy $^{13}$. » En complément de la notion de circularité, image de complétude et du tout, nous retrouvons aussi la figure de la

\footnotetext{
12 Montaigne, Les Essais, éd. Pierre Villey, coll. Quadrige, Paris, PUF, 1988, Liv. I, p. 183.

${ }^{13}$ Ibid., p. 188.
} 
symétrie. Cette dernière est introduite avec la citation d'Horace. Le corps d'une belle femme que finit une queue de poisson bafoue la symétrie naturelle de la figure humaine. C'est de symétrie dont il s'agit dans le rapport entre les grotesques de Montaigne et le tableau poli selon l'art de la Boétie. Le lecteur est entraîné, pour ainsi dire, dans une réalité qui se trouve au-delà du support matériel de l'œuvre, au-delà des propos, par ailleurs classiques, de Montaigne sur l'amitié.

Donc, par la perspective, notamment celle du dallage qui est la figure fondamentale chez Alberti pour établir une perspective correcte, par la pyramide visuelle et ses rayons, par la symétrie des espaces et des figures, l'œuvre est conçue en continuité avec le monde objectif et non détaché de lui. Il n'y a pas le monde réel et celui de l'illusion, le monde rationnel et celui de l'irrationnel; il n'y a qu'un seul monde parce que «All the world is a stage », parce que l'art est l'empreinte du monde. Dans A Midsummer Night's Dream, Oberon a aimé Hippolyta et la reine des fées fut autrefois amoureuse de Thésée. Si Puck demande à se faire pardonner c'est parce que la pièce montre que le monde passionnel et naturel est plus réel que le monde rationnel représenté par Thésée. La raison humaine est la véritable représentation de l'illusion comique comme de nombreux Humanistes ne cessent de le souligner à l'époque. Aujourd'hui, nous voyons encore que ce qui est appelé « réalité » est toujours plus incroyable que ce qui est appelé « fiction».

Ainsi, la fonction qu'Ernest Gilman attribue à la perspective dans le théâtre shakespearien, à savoir qu'elle suggère au spectateur de regarder de «biais» («awry») pour voir «juste» («a-right»), me semble insuffisante. En effet, implicitement, Gilman place le spectateur à l'« extérieur » de la pièce. De cette façon, il introduit une opposition entre «réalité » et «fiction» qui ne correspond pas à l'esprit du théâtre shakespearien où tout, au contraire, est mouvement, non seulement sur la scène mais aussi de cette dernière aux spectateurs. Comme dans la théorie albertienne, l'objectif du théâtre est de placer le spectateur au cœur de l'historia où chaque point de vue révèle une représentation et, par cela même, exclut l'autre, comme dans la toile d'Holbein. Par la multiplicité même des points de vue, c'est la notion d'infinité que Shakespeare suggère. L'intérêt de son théâtre, alors, n'est pas tant de le considérer comme «fiction », c'est-à-dire autonome par 
rapport à notre monde moderne mais l'empreinte du monde objectif, quelle qu'en soit l'époque.

En revanche, je vois dans la notion d'expérience, utilisée par Ernest Gilman, la clef du rapport entre le monde et sa représentation. Dans l'un comme dans l'autre il y a une architectonike, c'est-à-dire une structure, un sens, même si ce dernier reste flou dans l'appréhension. Ces deux structures sont liées par une correspondance symétrique. C'est de l'architectonike, dont la perspective est un élément fondamental, que l'œu vre d'art tire son statut de réalité. Cette dernière est supérieure à la réalité objective parce qu'elle est l'expression de l'Idée, d'un statut d'existence dont chacun ressent l'absence. Mieux structurée que la réalité quotidienne, l'œuvre d'art sollicite l'intelligence, la visio intellectualis, cette vue de l'esprit ("the mind's eye ») qui abolit les contraires constitutifs de ce monde. Donc, en dernier ressort, la réalité objective de la mimèsis se fonde sur la conscience morale. Shakespeare ne cesse de la questionner. C'est ce qui fait de son théâtre une expérience toujours vraie.

Jean-François CHAPPUIT Université de Versailles-Saint-Quentin-en-Yvelines 